\title{
Erratum to: Black Children in Hollywood Cinema
}

Erratum to:

Chapter 2 in: Debbie Olson, Establishing the Discourse of the Child DOI 10.1007/978-3-319-48273-6_2

Permission statement regarding the content of chapter 2 has been included.

The online version of the original chapter can be found under DOI 10.1007/978-3-319-48273-6_2

Debbie Olson

Department of English

Missouri Valley College

Marshall, Missouri, USA

Debbie Olson, Establishing the Discourse of the Child DOI 10.1007/978-3-319-48273-6_7

(C) The Editor(s) (if applicable) and The Author(s) 2017 